\title{
Perioperative Management of a Patient With Giant Axonal Neuropathy
}

\author{
Hiromi Kako a, b, d, Arlyne Thung ${ }^{\mathrm{a}, \mathrm{b}}$, Allan Beebe ${ }^{\mathrm{c}}$, Jan Klamar ${ }^{\mathrm{c}}$, Joseph D. Tobias ${ }^{\mathrm{a}, \mathrm{b}}$
}

\begin{abstract}
Giant axonal neuropathy (GAN) is a rare, autosomal recessive, progressive neurodegenerative disorder affecting both the central and peripheral nervous systems. The aberrant accumulation of cytoskeletalassociated proteins causes neurodegeneration resulting in both motor and sensory dysfunction. Given the invariable and progressive involvement of the neuromuscular system, anesthetic care is frequently needed to correct the orthopedic sequelae of the disorder. We present a 12-year-old girl with GAN who required anesthetic care for posterior spinal fusion to treat scoliosis. Previous reports of anesthetic care for patients with GAN are reviewed, and the perioperative considerations of these patients are discussed.
\end{abstract}

Keywords: Giant axonal neuropathy; Spinal fusion; Neuromuscular scoliosis; Neurodegenerative disorder

\section{Introduction}

First identified in 1972, giant axonal neuropathy (GAN) is a rare, autosomal recessive, progressive neurodegenerative disorder affecting both the central and peripheral nervous systems. Cytopathologically, the disorder is characterized by giant axons with derangements of the cytoskeletal components. The primary defect is a mutation in the GAN gene, which codes for the protein, gigaxonin. The defect in the gigaxonin system leads to the aberrant accumulation of cytoskeletal-associated proteins (neurofilaments) due to defective function of

Manuscript accepted for publication October 16, 2015

aDepartment of Anesthesiology and Pain Medicine, Nationwide Children's Hospital, Columbus, OH, USA

bepartment of Anesthesiology and Pain Medicine, the Ohio State University, Columbus, OH, USA

'Department of Orthopedic Surgery, Nationwide Children's Hospital and The Ohio State University Wexner Medical Center, Columbus, OH, USA

${ }^{d}$ Corresponding Author: Hiromi Kako, Department of Anesthesiology and Pain Medicine, Nationwide Children's Hospital, 700 Children's Drive, Columbus, OH 43205, USA. Email: Hiromi.Kako@Nationwidechildrens.org

doi: http://dx.doi.org/10.14740/jmc2346w the ubiquitin-proteasome system (UPS). The accumulation of these neurofilaments distends the axon leading to the both motor and sensory impairment with eventual neurodegeneration. The age of onset for GAN varies from soon after birth to 10 years of age. Clinical manifestations include both motor and sensory involvement resulting in poor coordination, muscle weakness, impaired sensation, absent tendon reflexes and gait disturbances. There is predominant distal involvement. The cranial nerves can also be affected, especially the oculomotor nerve and the facial nerve. Lesions in the brain and spinal cord can result in mental retardation, developmental delay, dysmetria, seizures, nystagmus and dysarthria, as well as signs of spasticity [1]. Affected children have a specific phenotypic facial appearance with curly hair. Treatment is symptomatic, and most patients are wheelchair-bound or die by the end of the second decade of life [2].

Given the invariable and progressive involvement of the neuromuscular system, anesthetic care is frequently needed during surgical procedures aimed at correcting the orthopedic sequelae of the disorder. We present a 12-year-old female with GAN who required anesthetic care for posterior spinal fusion. The perioperative considerations of such patients are reviewed, and the perioperative considerations of these patients are discussed.

\section{Case Report}

Institutional Review Board approval is not required for single case report at Nationwide Children's Hospital (Columbus, OH, USA). The patient was a wheelchair-bound, 12-year-old, 31.2 $\mathrm{kg}$ female with a diagnosis of GAN who was scheduled for posterior spinal fusion from T3 to L4 for scoliosis. Her other diagnoses included restrictive lung disease, mild obstructive sleep apnea (OSA), dysphagia causing aspiration of thin liquids and occasional choking at night due to secretions. The patient had been delivered at 39 weeks via cesarean section with a birth weight of 3,000 g. The patient initially did well, but began to show signs of abnormal gait and ataxia at approximately 5 years of age. At 6 years of age, she underwent sural nerve and muscle biopsy that identified the R242S mutation resulting in GAN. Preoperative physical examination revealed a cachectic girl with characteristic curly hair, extensive muscle atrophy and decreased muscle tone. Airway examination re- 
Table 1. Previous Reports of Anesthetic are for Patients With GAN

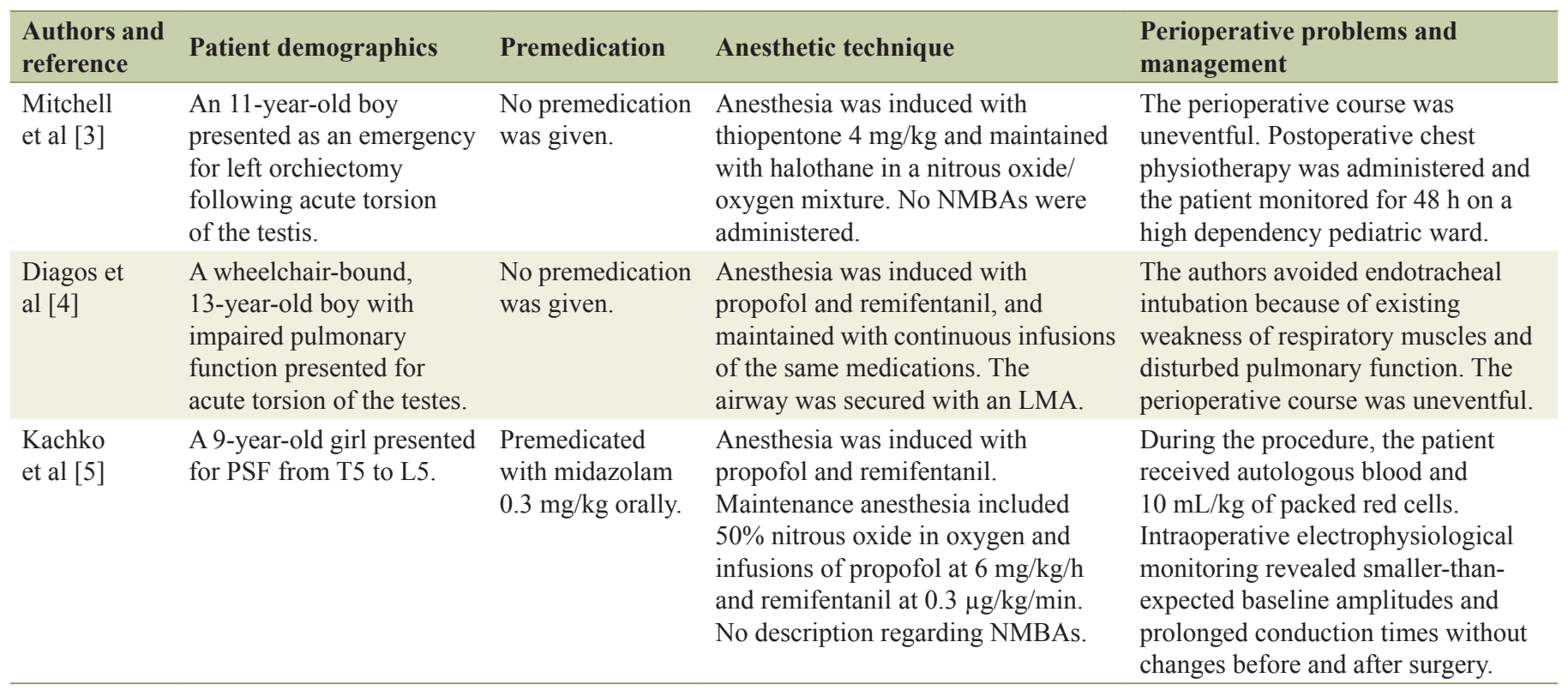

LMA: laryngeal mask airway; PSF: posterior spinal fusion.

vealed a Mallampati II view and moderate micrognathia with limited mouth opening. Preoperative pulmonary function tests revealed moderate restrictive lung disease with a forced vital capacity (FVC) of 52\% of predicted value and forced expiratory volume at $1.0 \mathrm{~s}(\mathrm{FEV} 1.0)$ at $52 \%$ of predicted value. Maximal inspiratory pressure (MIP) was $36 \mathrm{~cm} \mathrm{H}_{2} \mathrm{O}$, and maximal expiratory pressure (MEP) of $12 \mathrm{~cm} \mathrm{H}_{2} \mathrm{O}$ indicated respiratory muscle weakness. Preoperative sleep study revealed mild OSA. Preoperative echocardiogram and electrocardiogram were normal. Preoperative laboratory evaluation including complete blood count, renal function and coagulation function were normal. The patient was not on any medications preoperatively.

On the day of surgery, the patient was held nil per os for $6 \mathrm{~h}$ and transported to the operating room. No premedication was given preoperatively. Routine American Society of Anesthesiologists' monitors were placed and a 22 gauge intravenous cannula was placed in her left hand while she was inhaling 50\% nitrous oxide in oxygen. Anesthesia was induced with propofol $(80 \mathrm{mg}$ or $2.5 \mathrm{mg} / \mathrm{kg})$ and fentanyl $(25 \mu \mathrm{g}$ or $0.8 \mu \mathrm{g} /$ $\mathrm{kg}$ ). Bag-valve-mask ventilation was provided without difficulty. Neuromuscular blockade was provided with rocuronium $10 \mathrm{mg}(0.3 \mathrm{mg} / \mathrm{kg})$, and direct laryngoscopy was performed with a Miller 2 blade. Laryngoscopy revealed a Cormack and Lehane grade II-III view, and a $6.0 \mathrm{~mm}$ cuffed endotracheal tube (ETT) was placed on the first attempt with mild difficulty due to limited mouth opening using cricoid pressure and a stylet to direct the ETT under the epiglottis. Anesthesia was maintained with a propofol infusion $(100-160 \mu \mathrm{g} / \mathrm{kg} / \mathrm{min})$ which was titrated to maintain the bispectral index at $40-60$ and a remifentanil infusion $(0.1-1 \mu \mathrm{g} / \mathrm{kg} / \mathrm{min})$ to maintain the mean arterial pressure (MAP) at $55-65 \mathrm{~mm} \mathrm{Hg}$. After the induction of anesthesia, arterial access was obtained. Additionally, an 18 gauge intravenous cannula was placed in a right forearm vein.
A lactated Ringer's solution was provided at a maintenance rate. Tranexamic acid was administered for prevention of fibrinolysis $(50 \mathrm{mg} / \mathrm{kg}$ followed by an infusion of $5 \mathrm{mg} / \mathrm{kg} / \mathrm{h}$ ) and to limit intraoperative blood loss. The patient was turned and positioned prone. Baseline neurophysiological monitoring including transcranial electric motor evoked potentials (MEPs) and somatosensory evoked potentials (SSEPs) was obtained. Baseline SSEPs revealed poorly defined, unreliable and poorly reproducible waveforms. MEPs showed responses consistently from the brachioradialis muscles bilaterally. No significant changes occurred during the intraoperative monitoring period. During the surgical procedure, the MAP was maintained at 55 - $65 \mathrm{~mm} \mathrm{Hg}$ to minimize intraoperative blood loss. The surgical procedure lasted $4 \mathrm{~h}$ and included posterior spinal instrumentation and fusion from T3 to L4, utilizing titanium screws and cobalt chrome rods. Estimated blood loss was $200 \mathrm{~mL}$, and total intravenous fluids included $800 \mathrm{~mL}$ of isotonic crystalloid solution. No blood products or colloids were administered. Postoperative analgesia was provided by intravenous acetaminophen and hydromorphone titrated to respiratory rate prior to tracheal extubation. Following completion of the surgical procedure, the patient was transferred to the pediatric intensive care unit (PICU). Shortly after arrival to the PICU, per our usual practice for patients with respiratory compromise, her trachea was extubated to bi-level positive airway pressure support (BiPAP) at the settings of $16 / 8 \mathrm{~cm} \mathrm{H}_{2} \mathrm{O}$. Postoperative analgesia was provided with hydromorphone delivered via nurse-controlled analgesia. On postoperative day (POD) 1 , the BiPAP was discontinued in the morning, and she was transferred to the inpatient ward. Chest physiotherapy including cough assist was continued on the inpatient ward. The remainder of postoperative course was uncomplicated, and she was discharged home on POD 6. Physical therapy was continued at home. 


\section{Discussion}

As with the anesthetic care of all patients, the focus of effective perioperative care of patients with GAN begins with the preoperative examination and the identification of end-organ involvement by the primary disease process. Our search of the literature revealed only three anesthesia-related publications regarding the perioperative care of patients with GAN (Table 1) [3-5]. As noted in our patient and most patients with GAN, the potential anesthetic hazards include the potential for difficulties with airway management and endotracheal intubation, respiratory compromise related to restrictive pulmonary disease due to kyphoscoliosis and baseline muscle weakness, poor upper airway control with OSA, potential for aspiration and prolonged muscle weakness in response to medications.

The primary concern to anesthesia providers is the potential for difficult endotracheal intubation related to limited mouth opening and micrognathia as were noted in our patient. The three previous reports of anesthetic care for patients with GAN have not anecdotally noted problems with direct laryngoscopy and endotracheal intubation. Although we were able to successfully accomplish endotracheal intubation using direct laryngoscopy, the glottic view was less than optimal given the above-mentioned problems. Given these concerns, the ability to accomplish adequate bag-valve-mask ventilation should be demonstrated prior to the use of neuromuscular blocking agents (NMBAs). Additionally, the appropriate equipment for dealing with the difficult airway should be readily available including indirect laryngoscopy tools $[6,7]$.

In addition to the perioperative aspiration risk, poor airway tone and restrictive lung disease may predispose these patients to developing upper airway obstruction or respiratory failure during the postoperative period. Our patient had been previously diagnosed with OSA on polysomnography. These issues may be compounded by pre-existing respiratory dysfunction from hypotonia, poor cough effort, chronic aspiration or recurrent pneumonia. Preoperative assessment regarding the usual respiratory pattern, history of recurrent pneumonia or swallowing problems may identify at risk patients. Preoperative preparation should include aggressive treatment of respiratory infections and as cognitive function permits, instruction regarding the use of techniques such as incentive spirometry. Patients with pre-existing motor weakness and hypotonia may be sensitive to the effects of NMBAs, inhalational agents and sedative agents. There is an anecdotal report of prolonged muscle weakness following the administration of diazepam to a patient with GAN. The authors postulated that it was due to reduction of skeletal muscle tone at the spinal cord level by the benzodiazepine, not an effect at the neuromuscular junction [3]. On the other hand, premedication with midazolam was reported not to cause aggravation of muscle weakness [5]. As the residual effects of anesthetic agents may impact upper airway control and postoperative respiratory function, whenever feasible, short acting agents whose effects dissipate rapidly should be considered and careful titration of anesthetic agents is suggested with consideration of the monitoring of the depth of anesthesia for prolonged cases using the bispectral index. Two of the previous reports outlined the use of remifen- tanil and propofol infusions. In our patient, we also used a combination of remifentanil and propofol infusion to facilitate neuromonitoring [8]. General precautions include postoperative monitoring of respiratory function in an ICU setting, tracheal extubation when the patient is fully awake following the dissipation of the residual effects of intraoperative anesthetic agents, reversal of residual neuromuscular blockade and the judicious use of opioid analgesia. Extubation to non-invasive techniques of respiratory support such as BiPAP with a slow transition back to unassisted breathing may facilitate postoperative tracheal extubation and prevent atelectasis in patients with altered respiratory function like our patient $[9,10]$. Postoperatively, frequent periodic chest physiotherapy including assisted cough devices and incentive spirometry may also help to facilitate the clearance of mucus and prevention of postoperative respiratory complications.

Given the associated nerve and muscle involvement, one of the main perioperative concerns in providing anesthetic care of such patients is the choice of NMBAs, especially with regards to the safety of using succinylcholine [11]. Motor neuron involvement in GAN results in a decrease in nerve conduction and diminished acetylcholine release at the neuromuscular junction. Patients with a denervated neuromuscular junction may exhibit sensitivity to acetylcholine, succinylcholine and anticholinesterases. In the denervated muscle, the spread of acetylcholine receptors over the entire sarcolemma has been reported to lead to potassium efflux on depolarization following the administration of succinylcholine, resulting in potentially fatal arrhythmias related to hyperkalemia [12]. Given these concerns, it has been suggested that succinylcholine be avoided in patients with associated involvement of neuromuscular transmission $[12,13]$. On the other hand, the effect of non-depolarizing NMBAs in these patients has not been evaluated, and there is no consensus regarding the choice and dose of non-depolarizing NMBAs. In our patient, given that the surgical procedure including neurophysiological monitoring (MEPs and SSEPs), neuromuscular blockade needs to dissipate by the time of baseline neurophysiological monitoring, generally 20 - 30 min after anesthetic induction. The response to non-depolarizing NMBAs may be variable in each patient depending on the progression of the disease. Since there have been no reports regarding the use of nondepolarizing NMBAs in patients with GAN, we used a small dose of rocuronium $(0.3 \mathrm{mg} / \mathrm{kg})$ for endotracheal intubation [14]. Endotracheal intubation was easily managed with a small dose of rocuronium, and the neuromuscular blockade dissipated before the baseline neurophysiological examination. Alternatively, endotracheal intubation can also be accomplished using a combination of propofol and remifentanil without a NMBA $[15,16]$. While monitoring of neuromuscular function using train-of-four stimulation is generally beneficial, patchy loss of nerve function may limit the utility of peripheral nerve stimulation in assessment of recovery from neuromuscular blockade [3]. It may be optimal to identify a suitable site for monitoring and assess the baseline response prior to the administration of a NMBA.

As noted in our patient and one previous report, intraoperative electrophysiological monitoring can be challenging in neuromuscular scoliosis including patients with GAN. Intra- 
operative monitoring of SSEPs and MEPs is essential to assess the functional status of the dorsal sensory and ventral motor columns [17]. DiCindio et al investigated 68 children including 39 patients with neuromuscular scoliosis associated with cerebral palsy (CP) and 29 patients with non-CP related neuromuscular scoliosis for anterior and/or posterior spinal fusion during total intravenous anesthesia (TIVA) with propofol and sufentanil infusions [18]. In $30 \%$ of the patients with severe $\mathrm{CP}$ and $14 \%$ of non-CP neuromuscular scoliosis, SSEPs could not be obtained. Additionally, in $10 \%$ of severe CP and $14 \%$ of non-CP neuromuscular scoliosis, MEPs could not be obtained. Our patient's baseline SSEPs revealed poorly defined, unreliable and poorly reproducible waveforms without significant changes during the entire surgical procedure. The cause of poor SSEPs in our patient was unclear, but assumed to be related to the nature of disease process. However, MEPs showed consistent responses which were useful intraoperatively to monitor spinal cord integrity. Similar findings were reported previously in a patient with GAN who underwent posterior spinal fusion and neurophysiological monitoring [5]. The intraoperative electrophysiological monitoring showed the baseline amplitude of the waves to be smaller than expected with a prolonged conduction time. The authors recommended higher current stimulation and increasing the number of stimuli to overcome the low amplitude waves.

The choice of anesthetic agents will also impact the efficacy of neurophysiological monitoring. TIVA with a propofol infusion has been shown to have less of an effect on electrophysiological monitoring when compared to inhalational anesthetic agents including sevoflurane and isoflurane [19, 20]. Opioids have also been shown to cause only a modest decrease in the SSEP amplitude with a continuous infusion having less effect than bolus dosing [21]. Therefore, we chose a TIVA technique with propofol and remifentanil infusion for this case to optimize evoked potential monitoring while providing a short recovery time. Given its rapid metabolism, remifentanil allows for the provision of intense intraoperative analgesia without residual effects which might affect respiratory function during the postoperative period. When compared with a fentanyl infusion, remifentanil resulted in a quicker recovery and a shorter time to tracheal extubation with no significant difference in SSEP morphology [22]. Another consideration is a theoretical concern of the potential for malignant hyperthermia with inhalational anesthetic techniques in patients with neuromuscular disorders. Although the use of a volatile anesthetic-based technique has been anecdotally reported in a patient with GAN, there is no definitive data regarding this matter [3]. One case report stated that TIVA is a safe technique in patients with GAN [4].

In patients with GAN, autonomic nerve dysfunction results from neuronal damage from the accumulation of neurofilaments with the nerves of the autonomic nervous system [2]. Autonomic nerve dysfunction including impaired thermoregulation may impair intraoperative thermoregulation and predispose these patients to perioperative hypothermia. Hypothermia was noted in our patient with a nasopharyngeal temperature of $34.2^{\circ} \mathrm{C}$ despite active warming using a forced-air warming system, increasing the ambient temperature of the operating room, warming intravenous fluids, low fresh gas flow ventila- tion and the use of a humidifier throughout anesthetic induction and neurophysiological monitoring set-up times. Adverse physiological effects may become clinically relevant at a body temperature $\leq 36{ }^{\circ} \mathrm{C}$ including increased infectious complications, morbid cardiac events, coagulation disorders, prolonged length of hospital stay and increased costs. Hypothermia also enhances the effects of anesthetic agents resulting in prolonged recovery times and recovery room stay. Electrophysiological monitoring may also be affected with a decrease in SSEP amplitude [23]. Active intraoperative warming is imperative in patients with autonomic nerve dysfunction. Additionally, skin surface warming for 10 - 20 min immediately before anesthesia (pre-warming) has also been reported to minimize initial redistribution hypothermia and is recognized as the most effective means of preventing perioperative hypothermia [24, 25$]$.

Autonomic nervous system involvement may also predispose patients with GAN to an impaired response to hypotension [2]. Although there is no direct myocardial involvement, autonomic dysfunction may predispose these patients to perioperative hemodynamic instability. If the autonomic nervous system is affected, the degree of involvement is likely to be variable. Early in the disease, autonomic irritability may occur resulting in hypertension, tachycardia and arrhythmias in response to endogenous or exogenous catecholamines. With more advanced disease, cardiovascular reflexes may be impaired, resulting in an inability to respond to hypotension produced by blood loss, the administration of anesthetic agents, vasodilatation or intermittent positive pressure ventilation [3].

In summary, we present the perioperative management of a 12-year-old girl with GAN for posterior spinal fusion. As was noted in our patient and from anecdotal reports in the literature, several challenging problems may impact perioperative care. These include the potential for difficulties with airway management, autonomic dysfunction as well as restrictive pulmonary disease and OSA which may predispose to perioperative respiratory failure. A thorough preoperative examination with an assessment of end-organ impairment by the primary disease process, use of short-acting anesthetic agents to limit residual postoperative effects and close postoperative monitoring of respiratory function are suggested.

\section{Grant Support}

This case report was carried out without any grant or funding.

\section{Conflict of Interest}

No author has a conflict of interest with regard to this case report.

\section{References}

1. Yang Y, Allen E, Ding J, Wang W. Giant axonal neuropathy. Cell Mol Life Sci. 2007;64(5):601-609.

2. Gordon N. Giant axonal neuropathy. Dev Med Child 
Neurol. 2004;46(10):717-719.

3. Mitchell A, Moskovits PE. Anaesthesia for a patient with giant axonal neuropathy. Anaesthesia. 1991;46(6):469470.

4. Diagos P, Bos JA, Verrips A, Booij LH. Giant axonal neuropathy and anaesthesia. Anaesthesia. 2003;58(7):723724.

5. Kachko L, Tseitlin E, Straussberg R, Attias J, Bar-On E, Katz J. Anesthesia for a child with giant axonal neuropathy. Acta Anaesthesiol Scand. 2007;51(2):267-268.

6. Engelhardt T, Weiss M. A child with a difficult airway: what do I do next? Curr Opin Anaesthesiol. 2012;25(3):326-332.

7. Bryant J, Krishna SG, Tobias JD. The difficult airway in pediatrics. Advan Anesth. 2013;31:31-60.

8. Martin DP, Bhalla T, Thung A, Rice J, Beebe A, Samora W, Klamar J, et al. A preliminary study of volatile agents or total intravenous anesthesia for neurophysiological monitoring during posterior spinal fusion in adolescents with idiopathic scoliosis. Spine (Phila Pa 1976). 2014;39(22):E1318-1324.

9. Jaber S, Michelet P, Chanques G. Role of non-invasive ventilation (NIV) in the perioperative period. Best Pract Res Clin Anaesthesiol. 2010;24(2):253-265.

10. Pelosi P, Jaber S. Noninvasive respiratory support in the perioperative period. Curr Opin Anaesthesiol. 2010;23(2):233-238.

11. Martyn JA, Richtsfeld M. Succinylcholine-induced hyperkalemia in acquired pathologic states: etiologic factors and molecular mechanisms. Anesthesiology. 2006;104(1):158-169.

12. Kendig JJ, Bunker JP, Endow S. Succinylcholine-induced hyperkalemia: effects of succinylcholine on resting potentials and electrolyte distributions in normal and denervated muscle. Anesthesiology. 1972;36(2):132-137.

13. Azar I. The response of patients with neuromuscular disorders to muscle relaxants: a review. Anesthesiology. 1984;61(2):173-187.

14. Politis GD, Brill J, Jones J. Use of low-dose rocuronium for intubation of children during volunteer surgery abroad. Paediatr Anaesth. 2005;15(8):648-652.

15. Batra YK, Al Qattan AR, Ali SS, Qureshi MI, Kuriakose D, Migahed A. Assessment of tracheal intubating conditions in children using remifentanil and propofol without muscle relaxant. Paediatr Anaesth. 2004;14(6):452-456.
16. Politis GD, Tobias JD. Rapid sequence intubation without a neuromuscular blocking agent in a 14-year-old female patient with myasthenia gravis. Paediatr Anaesth. 2007;17(3):285-288.

17. Schwartz DM, Drummond DS, Schwartz JA, Wierzbowski LR, Sestokas AK, Pratt RE Jr, Turner LA. Neurophysiological monitoring during scoliosis surgery: a multimodality approach. Semin Spine Surg. 1997;9:97-111.

18. DiCindio S, Theroux M, Shah S, Miller F, Dabney K, Brislin RP, Schwartz D. Multimodality monitoring of transcranial electric motor and somatosensory-evoked potentials during surgical correction of spinal deformity in patients with cerebral palsy and other neuromuscular disorders. Spine (Phila Pa 1976). 2003;28(16):18511855; discussion 1855-1856.

19. Boisseau N, Madany M, Staccini P, Armando G, Martin F, Grimaud D, Raucoules-Aime M. Comparison of the effects of sevoflurane and propofol on cortical somatosensory evoked potentials. Br J Anaesth. 2002;88(6):785789.

20. Liu EH, Wong HK, Chia CP, Lim HJ, Chen ZY, Lee TL. Effects of isoflurane and propofol on cortical somatosensory evoked potentials during comparable depth of anaesthesia as guided by bispectral index. Br J Anaesth. 2005;94(2):193-197.

21. Pathak KS, Brown RH, Cascorbi HF, Nash CL, Jr. Effects of fentanyl and morphine on intraoperative somatosensory cortical-evoked potentials. Anesth Analg. 1984;63(9):833-837.

22. Samra SK, Dy EA, Welch KB, Lovely LK, Graziano GP. Remifentanil- and fentanyl-based anesthesia for intraoperative monitoring of somatosensory evoked potentials. Anesth Analg. 2001;92(6):1510-1515.

23. Seyal M, Mull B. Mechanisms of signal change during intraoperative somatosensory evoked potential monitoring of the spinal cord. J Clin Neurophysiol. 2002;19(5):409415.

24. Horn EP, Bein B, Bohm R, Steinfath M, Sahili N, Hocker $J$. The effect of short time periods of pre-operative warming in the prevention of peri-operative hypothermia. Anaesthesia. 2012;67(6):612-617.

25. Torossian A. Thermal management during anaesthesia and thermoregulation standards for the prevention of inadvertent perioperative hypothermia. Best Pract Res Clin Anaesthesiol. 2008;22(4):659-668. 